\title{
Nanoscale
}

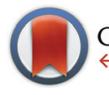

CrossMark \&lick for updates

Cite this: Nanoscale, 2016, 8, 2168

Received 27th July 2015

Accepted 17th December 2015

DOI: $10.1039 / c 5 n r 05032 d$

www.rsc.org/nanoscale

\section{Nanoscale mapping of heterogeneity of the polarization reversal in lead-free relaxor- ferroelectric ceramic composites $\uparrow$}

\author{
D. Gobeljic, ${ }^{a}$ V. V. Shvartsman, ${ }^{* a}$ A. Belianinov, ${ }^{b}$ B. Okatan, ${ }^{b}$ S. Jesse, ${ }^{b}$ S. V. Kalinin, ${ }^{b}$ \\ C. Groh, ${ }^{c}$ J. Rödel ${ }^{c}$ and D. C. Lupascu ${ }^{a}$
}

Relaxor/ferroelectric ceramic/ceramic composites have shown to be promising in generating large electromechanical strain at moderate electric fields. Nonetheless, the mechanisms of polarization and strain coupling between grains of different nature in the composites remain unclear. To rationalize the coupling mechanisms we performed advanced piezoresponse force microscopy (PFM) studies of 0.92BNT-0.06BT-0.02KNN/0.93BNT-0.07BT (ergodic/non-ergodic relaxor) composites. PFM is able to distinguish grains of different phases by characteristic domain patterns. Polarization switching has been probed locally, on a sub-grain scale. k-Means clustering analysis applied to arrays of local hysteresis loops reveals variations of polarization switching characteristics between the ergodic and non-ergodic relaxor grains. We report a different set of switching parameters for grains in the composites as opposed to the pure phase samples. Our results confirm ceramic/ceramic composites to be a viable approach to tailor the piezoelectric properties and optimize the macroscopic electromechanical characteristics.

\section{Introduction}

A strong piezoelectric effect and efficient electromechanical energy conversion are the key features of ferroelectric materials used in a large number of technological applications such as piezoelectric actuators, transducers, fuel injectors, micropositioning systems etc. ${ }^{1,2}$ The underlying reason for their wide applicability is polarization switching under an external electric field. In most of these materials, polarization switching is a complex process that includes nucleation of new domains and ferroelastic and ferroelectric domain wall motion. ${ }^{2,3}$ Furthermore, it is well know that the domain structure and domain movement affect the physical material properties, as dielectric permittivity, piezoelectric coefficient, ${ }^{4}$ hysteresis curve, ${ }^{5}$ aging, and fatigue. ${ }^{6}$

Nowadays $\mathrm{Pb}(\mathrm{Zr}, \mathrm{Ti}) \mathrm{O}_{3}$-based materials dominate the commercial market for applications of ferroelectrics. Nevertheless, due to global health and nature protection regulation acts, ${ }^{7}$

\footnotetext{
${ }^{a}$ Institute for Material Science and Center for Nanointegration Duisburg-Essen (CENIDE), University of Duisburg-Essen, Essen 45141, Germany.

E-mail: vladimir.shvartsman@uni-due.de

${ }^{b}$ Center for Nanophase Materials Sciences, Oak Ridge National Laboratory, Oak Ridge, TN 37830, USA

${ }^{c}$ Institute of Materials Science, Technische Universität Darmstadt, Darmstadt 64287, Germany

$\dagger$ Electronic supplementary information (ESI) available. See DOI: 10.1039/ c5nr05032d
}

there has been a growing trend to replace the environmentally harmful, lead-containing materials. ${ }^{8}$ This has triggered research activities worldwide to improve lead-free piezoelectrics. Among materials showing the best electromechanical performance are solid solutions from the ternary system $\mathrm{Bi}_{0.5} \mathrm{Na}_{0.5} \mathrm{TiO}_{3}-\mathrm{BaTiO}_{3}-\mathrm{K}_{0.5} \mathrm{Na}_{0.5} \mathrm{NbO}_{3}$ (BNT-BT-KNN). ${ }^{9,10}$ Moderate KNN doping yields a field-induced unipolar strain of $0.45 \%$ at $2 \% \mathrm{KNN}$ content. ${ }^{9}$ The structural origin of this giant strain is a reversible transformation between macroscopically cubic (relaxor) and non-cubic (ferroelectric) phases. ${ }^{11}$ Nonetheless, applicability of this material is restricted by the impractically high operational electric field $\left(\sim 8 \mathrm{kV} \mathrm{mm}^{-1}\right)$ and the large $P(E)$ hysteresis with a strong nonlinearity that is due to large dielectric losses in the material.

One of the ways to overcome these drawbacks is to consider families of the ceramic/ceramic composites consisting of a ferroelectric or non-ergodic relaxor phase (seed), embedded in a non-polar or ergodic relaxor phase (matrix). ${ }^{12}$ The presence of the ferroelectric phase with large polarization should enhance internal electric field strength in relaxor regions, compared to the average field applied to the entire sample. As a result, the induced phase transition in the ergodic relaxor phase occurs at smaller critical fields as opposed to the pure relaxor ceramic. In the past, several research groups have employed this approach. ${ }^{13-15}$ Studies of composites between the ergodic relaxor (ER) $0.92 \mathrm{BNT}-0.06 \mathrm{BT}-0.02 \mathrm{KNN}$ and the nonergodic relaxor (NE) 0.93BNT-0.07BT ceramics demon- 
strated a reduction of the critical field together with a relatively high value of the usable unipolar strain for materials with $10-30 \%$ of $0.93 \mathrm{BNT}-0.07 \mathrm{BT} .{ }^{15}$ Here, the terms ergodic and nonergodic refer to the phases that reversibly or irreversibly transform into a ferroelectric phase during electric field cycling. The experimental data has shown good agreement with numerical calculations ${ }^{15}$ leading us to believe that the strain level and the effective piezoelectric coefficient $d_{33}{ }^{*}$ are strongly governed by the ratio between BNT-7BT and BNT$6 \mathrm{BT}-2 \mathrm{KNN}$ content. Naturally, a change in the degree of ergodicity (by altering the $\mathrm{KNN}$ content) will affect the optimum volume content of the second phase. ${ }^{16}$

Nonetheless, there are questions with regard to the BNT-7BT/BNT-6BT-2KNN composites that are still open, particularly:

- which mechanism is responsible for the coupling between polarization in ferroelectric-like and relaxor grains of the composites, and

- how does the field-induced relaxor-ferroelectric phase transformation come about within the grains.

The internal electric field enhancement mechanism was proposed by Groh et al. ${ }^{15}$ Khansur et al. have suggested that the poling of relaxor grains is facilitated by the mechanical coupling between the grains within the inhomogeneous material structure. ${ }^{17}$ This suggestion has been corroborated by using multilayers of ergodic and nonergodic relaxors assembled in serial vs. parallel electric loading. However, the existence of the true composites with segregated constituents has not been convincingly shown. ${ }^{18}$ The key information on this issue can be obtained by a spatially resolved study of the field-induced polarization switching on a local scale comparable to the grain size.

Such a study is afforded by the piezoresponse force microscopy (PFM) technique. ${ }^{19}$ This technique, where high spatial resolution is achieved by using a sharp tip, allows for not only non-invasive domain imaging in polycrystalline materials, but also the manipulation of the polarization on the nanoscale and in situ monitoring of polarization switching. ${ }^{20}$

In this work, we present the PFM investigations of intragranular domain structures and the local polarization switching in ceramic/ceramic composites with varying volume fractions of the nominally nonergodic relaxor compound $0.93 \mathrm{Bi}_{0.5} \mathrm{Na}_{0.5} \mathrm{TiO}_{3}-0.07 \mathrm{BaTiO}_{3} \quad(\mathrm{BNT}-7 \mathrm{BT})$ in the ergodic relaxor $\quad 0.92 \mathrm{Bi}_{0.5} \mathrm{Na}_{0.5} \mathrm{TiO}_{3}-0.06 \mathrm{BaTiO}_{3}-0.02 \mathrm{~K}_{0.5} \mathrm{Na}_{0.5} \mathrm{NbO}_{3}$ (BNT-6BT-2KNN) matrix. Furthermore, we address the effect of the material composition on domain morphology and its homogeneity, as well as the effect of proximity of NE grains on polarization switching inside of ER grains.

\section{Experimental}

\section{Materials}

The polycrystalline ceramic/ceramic composites were prepared by mixing calcined $0.93 \mathrm{Bi}_{0.5} \mathrm{Na}_{0.5} \mathrm{TiO}_{3}-0.07 \mathrm{BaTiO}_{3}$ and $0.92 \mathrm{Bi}_{0.5} \mathrm{Na}_{0.5} \mathrm{TiO}_{3}-0.06 \mathrm{BaTiO}_{3}-0.02 \mathrm{~K}_{0.5} \mathrm{Na}_{0.5} \mathrm{NbO}_{3}$ powders.
Details of the powder preparation and ceramics sintering can be found elsewhere. ${ }^{16}$ The composite samples contained BNT7BT and BNT-6BT-2KNN in $0: 100,10: 90,30: 70$ and 100:0 vol\% ratios. The samples were prepared as $500 \mu \mathrm{m}$-thick pellets with a diameter of $10 \mathrm{~mm}$. For PFM measurements, the specimens were polished to optical quality using polycrystalline diamond paste (DP-Paste $\mathrm{P}$ by Struers $\mathrm{A} / \mathrm{S}$, Ballerup, Denmark) with abrasive particles of $15 \mu \mathrm{m}, 9 \mu \mathrm{m}, 3 \mu \mathrm{m}, 1 \mu \mathrm{m}$, and $0.4 \mu \mathrm{m}$ in size.

\section{Piezoresponse force microscopy}

The measurements were performed using a Cypher AFM (Asylum Research, Santa Barbara, CA, USA) equipped with an external high voltage amplifier and a custom-built BE controller. Data acquisition and system control were performed by custom-made LabView and MATLAB software. All measurements were performed using Pt/Ir coated cantilevers (PPP-EFM-W, Nanosensors) with a resonance frequency of approximately $75 \mathrm{kHz}$ and a force constant of about $2.8 \mathrm{~N} \mathrm{~m}^{-1}$. Domain imaging was performed using AC voltage with amplitude $V_{\mathrm{ac}}=2 \mathrm{~V}$, and frequency selected to be close to the contact resonant frequency of the cantilever. The resulting PFM images were analyzed using WSxM software. ${ }^{21}$ The local hysteresis loops were treated using custom developed MATLAB programs.

The description of principles of piezoresponse force microscopy can be found elsewhere. ${ }^{19,20}$ Briefly, PFM detects local electromechanical deformation of a sample induced by an AC voltage applied to the sample surface via a conductive PFM tip. The amplitude of the PFM signal is proportional to the local value of the longitudinal piezoelectric coefficient. The phase of the PFM signal depends on local polarization orientation: the phase of $0^{\circ}$ and $180^{\circ}$ correspond to the polarization direction parallel and antiparallel to the applied electric field, respectively.

To study polarization switching, we collected multiple local hysteresis loops over a 2D spatial grid using combined switching spectroscopy piezoresponse force microscopy (SS-PFM) and the band excitation (BE) approach. ${ }^{22}$ The local hysteresis loops were measured by applying a bipolar triangular waveform $\left(-V_{\max }<V<V_{\max }\right)$ formed by a sequence of short voltage pulses ( $4 \mathrm{~ms}$ ) of a constant $\mathrm{AC}$ amplitude besides a monotonically changing DC offset. DC voltage pulses were used for poling (writing), while the piezoresponse was read in-between two pulses (reading) at a DC bias of $0 \mathrm{~V}$. The loops were collected over a $40 \times 40$ point grid. At every grid point, six triangular waveforms with gradually increasing amplitude, $V_{\max }$, from $5 \mathrm{~V}$ to $45 \mathrm{~V}$ were applied. To reveal the regularity of polarization switching inside the grains with different domain patterns we utilized a $k$-means clustering algorithm.

\section{Results}

Fig. 1 provides typical amplitude and phase PFM images for the samples with different volume ratios between the BNT- 

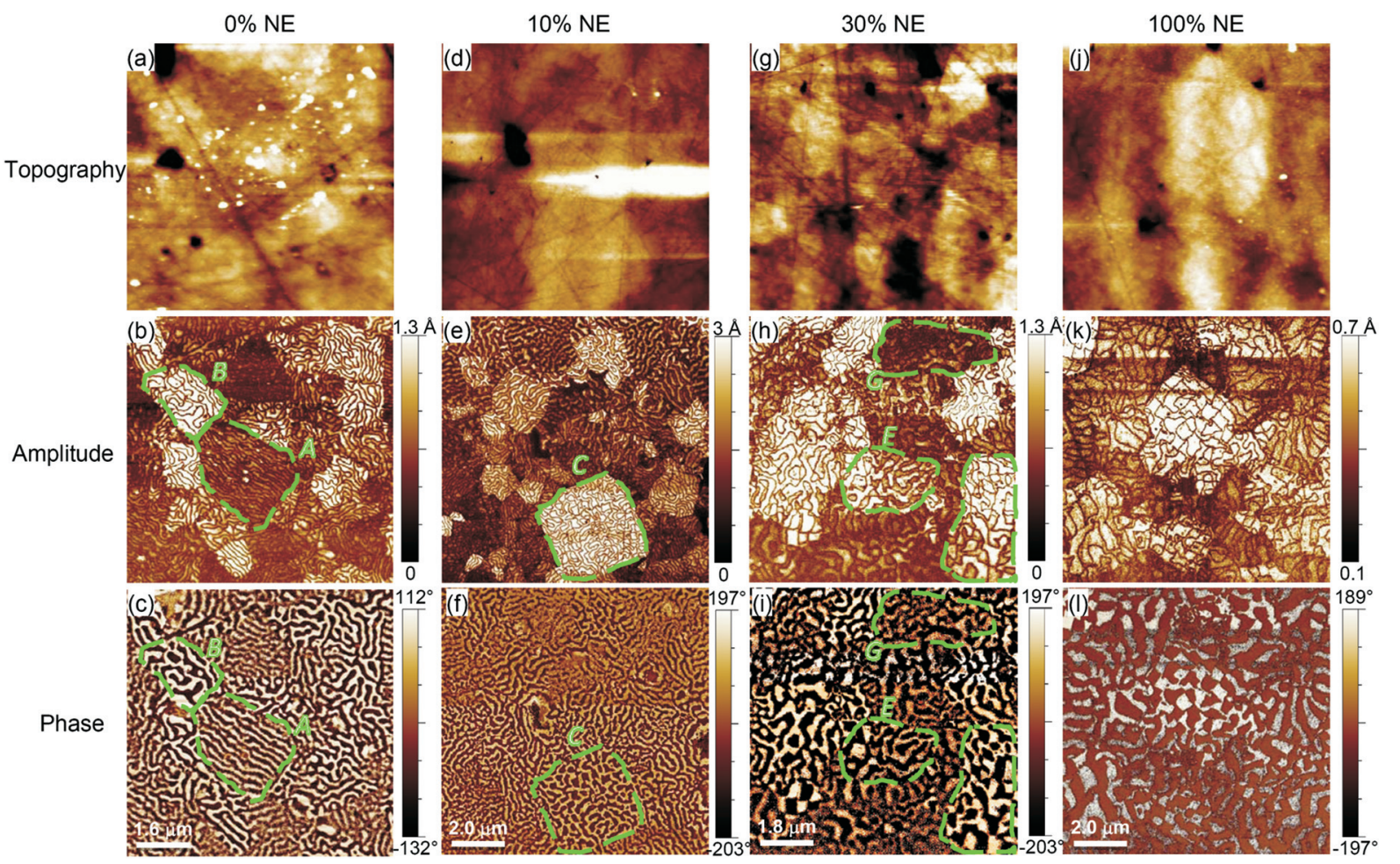

Fig. 1 Topography, amplitude, and phase PFM images of the BNT-6BT-2KNN/BNT-7BT ceramic/ceramic composites with different contents of nonergodic relaxor, BNT-7BT: 0\% (a-c), 10\% (d-f), 30\% (g-i), and 100\% (j-l).

7BT and BNT-6BT-2KNN constituents. Grains of different sizes, split into domains, are clearly seen. The amplitude signal represents the magnitude of the piezoresponse, and depends on the crystallographic orientation of the grain. The phase signal reflects the direction of the polarization vector. In Fig. 1, the bright color in the phase images corresponds to the "positive" polarization, where the polarization vector points up, normal to the surface. The dark regions represent domains with "negative" polarization which is directed down, into the bulk. The pure ergodic relaxor BNT-6BT-2KNN ( $0 \%$ NE) (Fig. 1(b and c)) features two types of domain patterns: disordered labyrinth-like and distorted stripe-like. The first type is typical for grains exhibiting a strong PFM response in the amplitude image (e.g. grain B in Fig. 1(b)). The second type of domain, by contrast, is characteristic for grains with a weak PFM signal, as demonstrated for grain A. According to electron backscatter diffraction data (Fig. S1, ESI $\dagger$ ), the stronger PFM signal corresponds to grains with crystallographic orientation close to $\langle 001\rangle_{c}$ directions, whereas the grains with quasi-stripe domains have orientation close to $\langle 111\rangle_{c}$ directions and show a weaker piezoresponse. The domain structure analysis demonstrates that the labyrinth domain patterns have a characteristic width of 100-150 $\mathrm{nm}$ within a single grain.

The domain structure of the sample with $10 \%$ of the NE (Fig. 1(e and f)) displays no drastic changes in comparison with the $0 \% \mathrm{NE}$ sample. Both the labyrinth-like and quasistripe features are observed over the entire sample area. Weak reordering of the domains occurs which is demonstrated as the shortening of labyrinth domain length without their broadening, as in grain labeled $\mathrm{C}$.

A further increase of the BNT-7BT content to $30 \mathrm{vol} \%$ $(30 \%$ NE) notably influences the domain morphology (Fig. 1(h and i)). Particularly, a new type of domain structure having a checkerboard pattern appears (see grain in the bottom left corner). These patterns are characterized by a larger domain size than those observed for the labyrinth structures. Besides, in many grains (e.g. grains $\mathrm{E}$ and $\mathrm{G}$ ) the labyrinth patterns are still observed. We note that the average domain size increases as compared to the samples with less of the NE content.

Finally, BNT-7BT (100\% NE) demonstrates mainly the checkerboard domain patterns (Fig. 1(k and l)). Within some grains, stripe domains are also observed, but the pattern is more regular along with the transverse dimension larger than in the pure relaxor sample. The average domain size for the $100 \%$ NE sample is an order of magnitude larger than that of the $0 \%$ NE sample described in Fig. 1(b and c). Similar to the $0 \%$ NE sample, the strongest and the weakest PFM signals are typical for $\langle 001\rangle_{c}$ - and $\langle 111\rangle_{c}$-oriented grains, respectively.

To study the local polarization switching behavior with respect to the content of nonergodic BNT-7BT we collected multiple local PFM hysteresis loops over a 2D spatial grid. 1600 local hysteresis loops were collected over the $5 \mu \mathrm{m} \times 5 \mu \mathrm{m}$ scan areas for each sample. Characteristic parameters of local polarization switching were addressed on several length scales:

(a) average switching behavior of each composite sample (compositional diversity); 
(b) switching behavior on the single grain scale; assuming that the characteristic labyrinth or checkerboard domain morphologies correspond to the ergodic or the nonergodic relaxor constituents, respectively (for more details see the Discussion section) (constituent diversity);

(c) switching behavior inside an ER grain depending on the distance from the boundary to the neighboring NE grain (proximity effect).

The hysteresis loop parameters of interest are the maximum piezoresponse, $D_{\max }$, remanent piezoresponse at zero bias voltage, $D_{0}$, coercive voltage, $V_{0}$, and the work of switching, $A_{\mathrm{s}}$.

\section{Compositional diversity}

Fig. 2 illustrates the effective (spatially averaged over the entire switching grid) hysteresis loops for all four samples. The presented loops correspond to the last switching cycle with the maximum amplitude of the DC voltage. The variation in the switching behavior as the amount of the NE component in the composites changes can be clearly observed. The 100\% NE sample shows a typical ferroelectric loop that is widely open, symmetric and has a very strong piezoresponse. As the content of the NE component drops to $30 \mathrm{vol} \%$, both $D_{\max }$ and $D_{0}$ decay strongly. For the $10 \%$ NE sample, the average loop shows a slim hysteresis and further decrease of the remanent piezoresponse. Fig. 3(a) reveals an almost linear decay in the remanent piezoresponse with increasing ER content. Interestingly, although $D_{\max }$ diminishes in the average loop for the pure ergodic relaxor, the piezoresponse remains almost constant as the DC bias drops from the maximum value of $45 \mathrm{~V}$ to zero. Fig. 3(b) compares the coercive voltage and the hysteresis area for different samples. Note that the voltage required to perform switching increases with increasing NE content and is maximized for the $30 \% \mathrm{NE}$ composite. The loop area, i.e. the work of switching $A_{\mathrm{s}}$ also grows as the content of BNT-7BT increases and is much larger for the pure NE sample.

The averaged loops show an asymmetry relative to both piezoresponse and bias voltage axis. The asymmetry of the

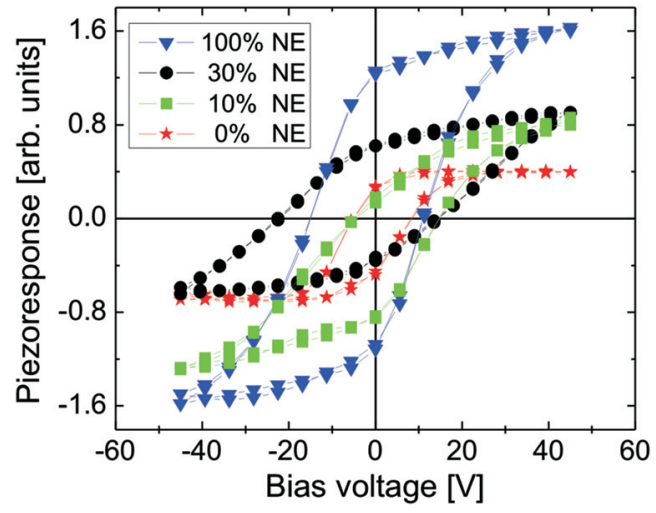

Fig. 2 Compositional diversity: spatially averaged local hysteresis loops for the composites with different contents of BNT-7BT phase.
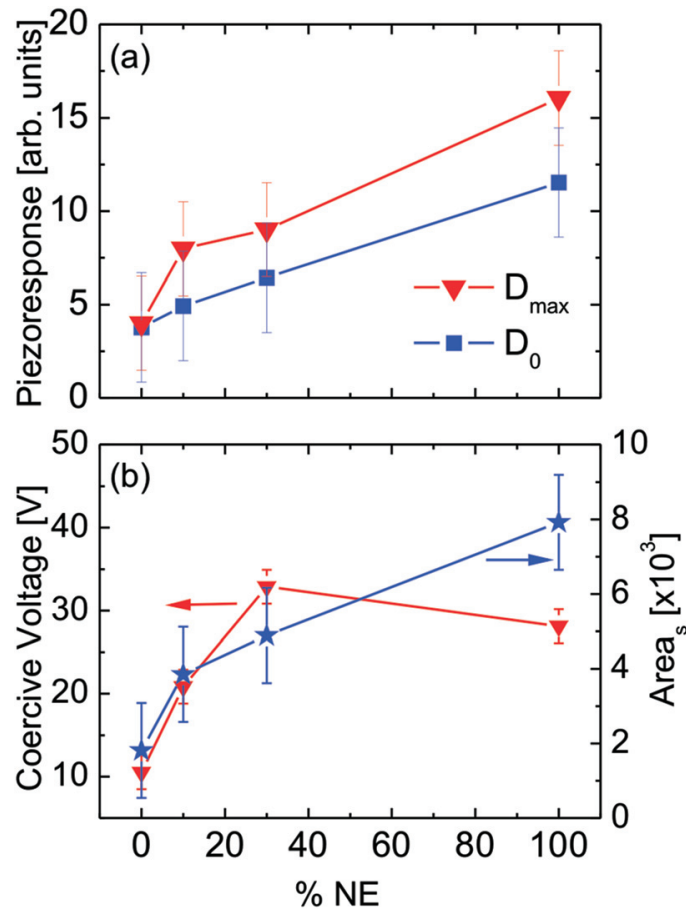

Fig. 3 Compositional diversity: averaged hysteresis loop parameters: (a) maximum and remanent piezoresponse, (b) coercive voltage and work of switching as a function of the BNT-7BT content. Shown data points correspond to a mean between positive and negative values for each hysteresis loop averaged over the entire grid. The error bars are the standard deviation from the mean value.

PFM hysteresis loops has been often reported for thin films as well as for bulk samples. ${ }^{23-25}$ Typically, it is related to the existence of built-in internal fields that make one polarization state energetically more preferable. The nature of these fields can be different. Liu et al. suggested that in relaxors these are random electric fields resulting from random distribution of cations with different valencies over equivalent crystallographic positions. ${ }^{24}$ Also charged defects, e.g. oxygen or potassium vacancies, accumulated in a subsurface layer, can be sources of the internal field. In such a case, the field is unipolar and can induce the asymmetry of the loops in one direction. Fig. 2 shows the spatially averaged hysteresis loops. Therefore, the observed asymmetry indicates the dominance of a certain polarity of the internal fields. A detailed analysis of the origins of this asymmetry demands studies on defect distributions in our samples and is out of scope of the presented research.

\section{Constituent diversity}

Based on domain imaging, the characteristic domain morphology is assumed to be ruled by the material content. Therefore, we expect that the local polarization switching also correlates with the composition of a single grain. To check this, the switching behavior was studied on the level of a single grain using the $k$-means clustering analysis. 
$k$-Means clustering (KMC) is one of the most frequently used clustering approaches, applied in pattern recognition and classification, ${ }^{26}$ data mining and knowledge discovery, ${ }^{27,28}$ as well as in data compression and vector quantization. KMC aims to partition $n$ observations into $k$ clusters in which each observation belongs to the cluster with the nearest mean value based on a specified distance metric between the data points in the $n$-dimensional space. In our case, KMC was applied to analyze local hysteresis loops obtained by PFM using MATLAB. The criterion by which KMC identifies, distinguishes, and plots the map of clusters was the hysteresis loop shape. KMC tracked over the $40 \times 40$ switching points, grouped them into 10 different clusters $(k=10)$, and generated the corresponding cluster maps.

Fig. 4 summarizes the results of $k$-means clustering analysis for the $30 \%$ NE sample. In a cluster map, the points belonging to the same cluster are indicated by using the same colour. For each cluster recognized by KMC a spatial map of points belonging to that cluster and a representative hysteresis loop were generated. The PFM image is also outlined for the sake of comparison. As already mentioned, KMC groups the local PFM loops into 10 individual clusters; however a few of them contain just a few points and were not considered in further analyses.

(a)

(b)
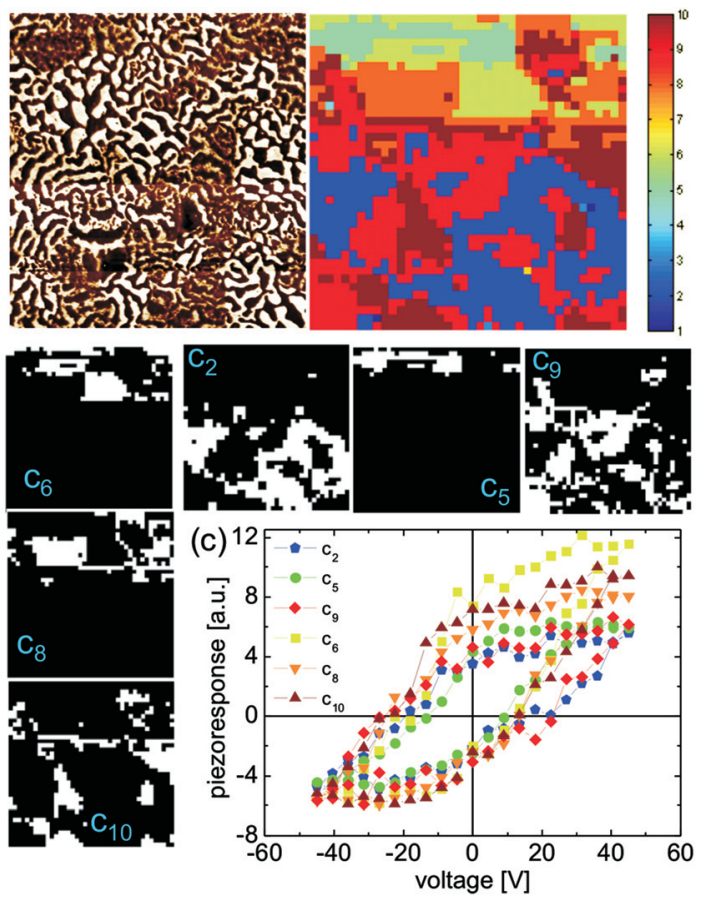

Fig. 4 Constituent diversity: (a) the PFM image and the corresponding $k$-means clustering map for polarization switching for the composite ceramic with 30 vol\% of BNT-7BT phase. 10 clusters are considered. The points belonging to the same cluster have the same color in the cluster map. Solid lines in the PFM image mark the grain boundaries; (b) The maps for clusters showing ergodic-like switching behavior $\left(c_{2}, c_{5}\right.$ and $c_{9}$ ) and for clusters within which the ferroelectric-like hysteresis loops are obtained $\left(c_{6}, c_{8}\right.$, and $\left.c_{10}\right)$; (c) the local hysteresis loops of $c_{i}$ clusters.
Fig. 4(c) compares the representative loops for clusters that occupy the major part of the studied region. The representative loops can be separated into two groups. For clusters $\mathrm{c}_{6}, \mathrm{c}_{8}$, and $\mathrm{c}_{10}$, the loops show ferroelectric-like features with a large maximum and remanent piezoresponse. Switching is successfully performed in both directions, even though the full loop saturation is not achieved for the maximum applied voltage. There is a relatively large difference between $D_{\max }$ and $D_{0}$. For approximately the same coercive voltages, a noticeably weaker piezoelectric activity is observed for clusters $c_{2}, c_{5}$, and $c_{9}$. A relatively low value of the maximum piezoresponse, that is, a lower effective piezoelectric coefficient $\left(d_{33}\right)_{\text {eff }}$ and smaller work of switching, is noted (Fig. 4(c)). By comparing with the phase PFM image (Fig. 4(a)) one can see that the first type of loop (clusters $c_{8}$ and $c_{10}$ ) is mainly associated with grains showing the checkerboard domain pattern (presumably with dominant NE composition), while the second one (clusters $\mathrm{c}_{2}$ and $c_{9}$ ) is related to grains having a labyrinth structure (ER grains). Nevertheless, intermixing of the composite constituents might appear, particularly in the vicinity of grain boundaries. The comparison of PFM and KMC maps reveals that some grains consist of several regions belonging to different clusters (Fig. 4(b)). E.g. for some grains with the checkerboard domain pattern peripheral regions show the suppressed hysteresis loops (compare clusters $c_{8}$ and $c_{9}$ for the grain in the top left corner and clusters $c_{10}$ and $c_{2}$ for the grain at the right side of the scan).

It is interesting that KMC does not show a substantial variety of the local polarization switching behavior for the parent constituents, $100 \% \mathrm{NE}$ and $0 \% \mathrm{NE}$ samples. The clusters can still be distinguished, and the representative local loops reveal a uniform shape to some extent (see details in Fig. S2 and S3, ESI $\dagger$ ). Also the sample with $10 \%$ of NE phase shows a less variety in the local loop shape between clusters (see Fig. S4, ESI $\dagger$ ). For most of the clusters, the representative loops have a strong negative offset. Only for two grains, a positive offset is observed. However, no correlation between the polarity of the offset and domain pattern can be established. Dominance of the local loops with the negative offset results in stronger asymmetry of the average loop for the sample with $10 \%$ of NE phase as compared to other samples.

\section{Proximity effect}

This approach allows for investigation of the influence of nonergodic grains on the properties of the surrounding ergodic relaxor matrix. At this length scale, polarization switching behavior in a "labyrinth-like" grain is analyzed as a function of distance from a next "checkerboard" grain. The loops were collected at positions marked in Fig. 5(a). For better statistical accuracy, we performed averaging over loops over a "narrow" $5 \times 1$ grid around the marked locations. The switching parameters inside the "checkerboard" grain (position 1) are plotted for comparison. In accordance with the constituent and compositional diversity, the largest $D_{\max }$ and $D_{0}$ values are observed inside the NE grain (Fig. 5(b)). For the ER grain, a drop of both $D_{0}$ and the loop area, $A_{\mathrm{s}}$, (Fig. 5(c)) is 
(a)

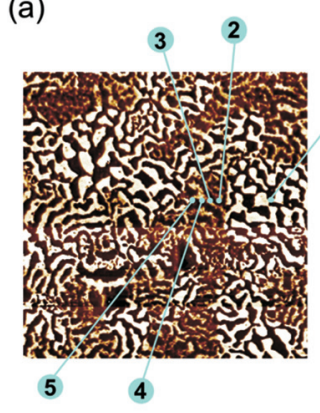

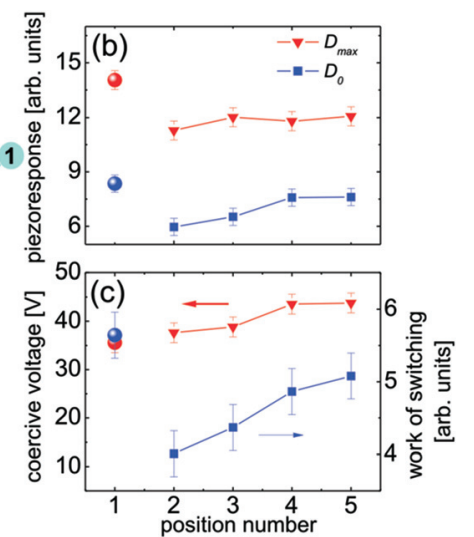

Fig. 5 Proximity effect: (a) the PFM image of the composite ceramic with 30 vol\% of BNT-7BT phase. Variation of the hysteresis loops parameters: maximum and remanent piezoresponse (b), coercive voltage and work of switching (c) inside an ER grain (triangles and squares). As a reference the hysteresis loops parameters inside the NE grain are shown (circles). The positions are indicated in the panel (a).

observed by moving off the boundary with the NE grain on the left. At the same time, the coercive voltage and maximal piezoresponse, $D_{\max }$, are almost independent of the position inside the ER grain. It has to be mentioned that the ER grain on its left side borders upon another 'checkerboard' grain. Therefore, assuming that the local switching in the ER grain is affected by the proximity to a NE grain, a similar behavior should be expected for the outermost relaxor sites (2 and 5), which is not the case.

\section{Discussion}

The PFM data are discussed in context of the local switching properties providing information on the microscopic mechanisms that can govern the reported macroscopic high electromechanical strain in the ceramic/ceramic composites. ${ }^{15}$

So far, the ceramic/ceramic composites studied within this work have been investigated by different approaches, from macroscopic strain over in situ high-energy X-ray diffraction to energy dispersive $\mathrm{X}$-ray spectroscopy and transmission electron microscopy measurements. ${ }^{15-18}$ The macroscopic measurements reported that the high unipolar strain in these materials can be achieved at relatively low electric fields and that the maximum strain level strongly depends on the amount of the BNT-7BT constituent. ${ }^{15}$ Composite behavior modeling showed that the tangled ergodic and nonergodic phases allow tailoring of the electromechanical properties of the high-strain materials, assuming the field-induced strain to be facilitated by the NE component that is surrounded by the relaxor phase. ${ }^{16}$ The structural measurements ${ }^{17}$ confirm the inhomogeneity of the structure that is pseudocubic at the zero field state, and experience either irreversible or reversible phase transition under the applied electric field depending on the volume percentage of the non-ergodic component in the material.

Our PFM studies have provided a new insight into the functional inhomogeneity of the materials by addressing the polarization and electromechanical behavior of the constituent phases separately.

Comparing the dominant domain morphologies observed in the two parent compositions ( $0 \% \mathrm{NE}$ and $100 \% \mathrm{NE})$, we can attribute the checkerboard domain pattern to the BNT-7BT phase. The data regarding the crystalline structure and the symmetry of this composition are controversial. For the unpoled state, the symmetry of BNT-7BT is usually reported as pseudocubic, but under a sufficiently high electric field it irreversibly transforms into an MPB between the R3c and P4 mm phase. ${ }^{29}$ Nonetheless, the strong PFM response indicates that the local symmetry is not cubic even in the pristine state, at least in a subsurface layer probed by PFM. At the same time, the characteristic checkerboard pattern can be an indication of the local tetragonal symmetry. ${ }^{30}$

We found no grains with the checkerboard pattern in the BNT-6BT-2KNN parent composition, for which the labyrinth and distorted stripe patterns are typical. A similar domain morphology has been reported for other relaxor compounds. ${ }^{31,32}$ Static domain patterns and a relatively large piezoresponse indicate that the ergodicity of the bulk relaxor state is broken in a sub-surface volume probed by PFM. Based on the results of X-ray diffraction ${ }^{33}$ and PFM measurements, ${ }^{31,32,34}$ different groups reported the existence of a ferroelectric-like surface layer for materials, which show macroscopically relaxor behavior. It has been suggested that in "cubic" relaxors with the perovskite structure the surface breaks the symmetry between possible polarization orientations in PNRs due to elastic interactions. This results in a transition from the cubic phase to a tetragonal phase. ${ }^{32}$ The electrical interaction results in secondary breaking of symmetry in plane and the formation of domains with antiparallel polarization. ${ }^{32}$ The equilibrium domain configurations are predefined by the system energetics, yielding the characteristic domain size. A similar scenario can be adopted for the studied materials.

In our opinion, the internal fields responsible for the asymmetry of the local hysteresis loops cannot drive the formation of this ferroelectric like state. Otherwise, a unipolar or strongly biased PFM signal distribution would be expected, which actually is not the case.

The transformation from regular to irregular domain patterns has been reported for systems showing a crossover from ferroelectric to relaxor behavior ${ }^{31}$ and agrees well with the more relaxor character of BNT-BT-KNN phase. Besides, the change of the characteristic domain size is an indication of a different composition. Due to the comparable grain size of the BNT-BT and BNT-BT-KNN samples, the size effect on the domain pattern can be excluded.

For the sample with $30 \%$ of BNT-7BT, a coexistence of grains with the domain patterns characteristic for both the parent compositions confirms the formation of the composite 
with two separate phases. Nevertheless, some intermixing of the constituents, especially close to the grain boundaries, is not excluded.

The average polarization switching behavior of the studied samples (compositional diversity) reveals a gradual evolution from tilted, slim hysteresis loops typical for the ergodic relaxors $^{35}$ to those with large hysteresis and a high piezoresponse characteristic for the samples with increased BNT-7BT content (Fig. 2). While the ergodic relaxor behavior is broken in the regions probed by PFM, some features typical for the relaxor state, like fast decay of the field induced piezoresponse and zero (or even) negative stability gap ${ }^{36}$ are retained in the BNT$6 \mathrm{BT}-2 \mathrm{KNN}$ sample. The nonergodic BNT-7BT phase features a larger average piezoresponse and more stable remanent polarization (the stability gap is positive), which is in agreement with the macroscopic behavior.

The averaged PFM hysteresis loop of $30 \%$ NE sample apparently reveals an intermediate behavior due to the contributions from both phases. For this sample, the $k$-means analysis distinguishes between the switching behavior in grains belonging to two different phases of the composite (constituent diversity) (Fig. 6). Again, the "ER" grains with the labyrinth pattern exhibit more tilted hysteresis loops and smaller piezoresponse than the "NE" ones (Fig. 6). Note that these "ergodic" grains exhibit broader hysteresis loops as compared to the average hysteresis loop collected at a larger scale for the $0 \% \mathrm{NE}$ sample. This can be an indication that the state of BNT-6BT$2 \mathrm{KNN}$ grains in the composite is altered by the BNT-7BT phase, which can be present not only as macroscopic grains, but also in the form of nanometer-sized inclusions distributed in the surrounded "ergodic relaxor" matrix. Such ferroelectriclike inclusions have been mentioned to often arise in between or within ubiquitous relaxor grains in the studied composites. ${ }^{17}$ On the one hand, they cannot be detected by PFM in the scanning mode due to their small size and the low probing

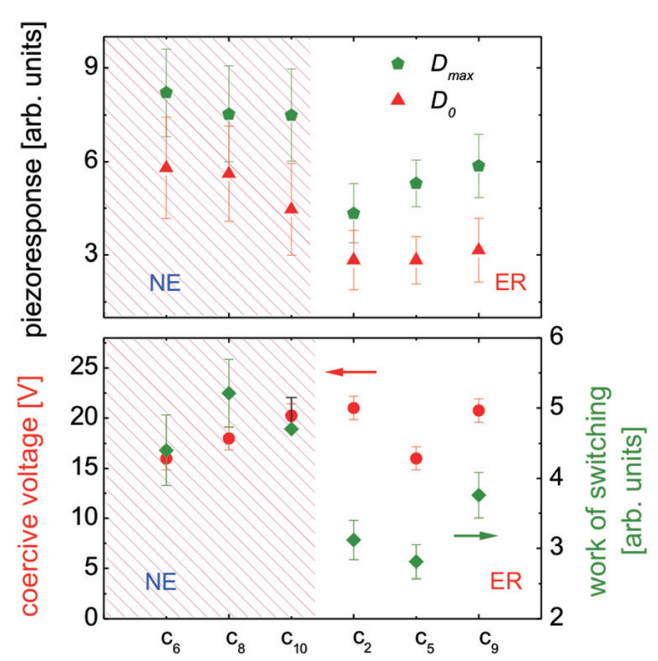

Fig. 6 Comparison of the maximum piezoresponse $D_{\max }$ remanent piezoresponse $D_{0}$, and coercive voltages of the different switching clusters, $c_{i}$.
AC voltage that is not high enough to trigger their growth. On the other hand, in the spectroscopic mode (switching experiments) sufficiently high DC bias voltage may provoke the expansion of these inclusions into bigger ferroelectric domains that contribute to the larger electric field-induced PFM signal in comparison with the pure ergodic 0\% NE composition. After the bias removal, these domains remain stable contributing to the remanent piezoresponse $D_{0}$, while the "ergodic relaxor" portion of the grain reverses.

Comparing the switching behavior at different locations inside the "ergodic relaxor" grain, we did not find an unambiguous correlation between the switching parameters and distance from the interface with the neighboring "nonergodic" grain. One can see, however, the variations of both the maximum piezoresponse and the switching work through the grain. This can be assigned e.g. to the variation of local mechanical stress or to the composition gradients due to a limited inter-diffusion between the NE and ER phases. ${ }^{18}$

In light of tailoring the electromechanical response of studied materials, it is important to discuss how the properties of constituents change in the composites. To this extent, we have compared the experimental data obtained on different scales. For convenience, we consider the compositional diversity as a macroscopic scale approach, while the proximityeffect data is discussed in the context of a single-point scale.

By contrasting the results obtained at these two scales, one can note that for the ergodic phase of the composite sample, the maximum piezoresponse on the single-point scale is strongly improved as compared to the pure BNT-6BT-2KNN sample. This can be attributed to the effect of the neighborhood of the BNT-7BT grains. The loop area analysis additionally supports this idea: the work of switching for the farthest position inside the ER grain is not as low as in the $0 \% \mathrm{NE}$ sample, while not as high as obtained in the pure NE ceramics. The influence of the two neighboring phases is mutual. While on the single-point scale the NE grains in the composite exhibit a larger work of switching than the ER grains (similar to the largest switching work, i.e. switching losses, on the macroscopic scale for the $100 \%$ NE ceramics between all the studied samples), when comparing these two values it appears that the switching losses are higher on the macroscale. It should also be mentioned, that the interior sites of the ER grains in the composite exhibit properties that are similar to the properties of the $30 \%$ NE sample as a whole. This indicates that the average response of the $30 \%$ NE sample can be considered as a collective effect of the structure that is inhomogeneous already on the grain length scale. Thus the grains of a different phase can be incorporated into the microstructure for fine tuning of the piezoproperties on the macroscale.

\section{Conclusions}

Piezoresponse force microscopy studies confirmed the formation of the ceramic/ceramic BNT-6BT-2KNN/BNT-7BT 
composites. Grains of the BNT-6BT-2KNN phase feature labyrinth and distorted stripe domain patterns characteristic of relaxors, while the BNT-7BT grains typically exhibit more ordered checkerboard domain morphology. The ergodic relaxor character of bulk BNT-6BT-2KNN seems to be broken at the surface, which is similar to the observations in other relaxors. Nevertheless, a different polarization switching behavior between nominally ergodic and nonergodic constituents was observed both on the macroscopic averaged scale and between different grains in the composite ceramic with $30 \%$ of BNT-7BT. We observed an improvement of the piezoelectric response of $\mathrm{BNT}-6 \mathrm{BT}-2 \mathrm{KNN}$ grains in the composite in comparison with the single phase ceramics. However, no pronounced effect of proximity to a BNT-7BT grain on the local polarization switching in the "ergodic relaxor" grains was found. This can be related to the conditions of the used approach. Indeed, the local hysteresis loops were probed successively at different locations. Contrary to the macroscopic measurements, in our experiment the switching in "ergodic relaxor" grains occurs rather independently from that in "nonergodic" grains. Therefore, the influence of the more easily switched grain on the harder switched neighborhood is less pronounced.

\section{Acknowledgements}

This work has been supported by the European Commission within FP7 Marie Curie Initial Training Network "Nanomotion" (grant agreement no. 290158). The research was conducted at the Center for Nanophase Materials Sciences, which is sponsored at Oak Ridge National Laboratory by the Scientific User Facilities Division, Proposal Number: CNMS2013-250. DG acknowledges the experimental support of Dr Evgeni Strelcov. CG was supported by the Leibniz program of the Deutsche Forschungsgemeinschaft under Ro 954/22.

\section{Notes and references}

1 K. Uchino, J. Electroceram., 2008, 20, 301.

2 W. Heywang, K. Lubitz and W. Wersing, Piezoelectricity: Evolution and Future of a Technology, Springer, Berlin Heidelberg, Germany, 2008.

3 A. Tagantsev, L. E. Cross and J. Fousek, Domains in Ferroic Crystals and Thin Films, Springer, New York, USA, 2010.

4 V. Nagarajan, A. Roytburd, A. Stanishevsky, S. Prasertchoung, T. Zhao, L. Chen, J. Melngailis, O. Auciello and R. Ramesh, Nat. Mater., 2003, 2, 43.

5 G. Arlt, Ferroelectrics, 1990, 104, 217.

6 G. Arlt, Ieee 1990 Ultrasonics Symposium: Proceedings, 1990, 2, 733.

7 EU-Directive 2011/65/EU, Restriction of the use of certain hazardous substances in electrical and electronic equipment (RoHS), Off. J. Eur. Union, 2011, L174, 88.
8 J. Rödel, K. G. Webber, R. Dittmer, W. Jo, M. Kimura and D. Damjanovic, J. Eur. Ceram. Soc., 2015, 35, 1659.

9 S.-T. Zhang, A. B. Kounga, E. Aulbach, H. Ehrenberg and J. Rödel, Appl. Phys. Lett., 2007, 91, 112906.

10 J. Rödel, W. Jo, K. T. P. Seifert, E. M. Anton, T. Granzow and D. Damjanovic, J. Am. Ceram. Soc., 2009, 92, 1153.

11 W. Jo, T. Granzow, E. Aulbach, J. Rödel and D. Damjanovic, J. Appl. Phys., 2009, 105, 094102.

12 D. S. Lee, D. H. Lim, M. S. Kim, K. H. Kim and S. J. Jeong, Appl. Phys. Lett., 2011, 99, 062906.

13 S.-Y. Choi, S.-J. Jeong, D.-S. Lee, M.-S. Kim, J.-S. Lee, J. H. Cho, B. I. Kim and Y. Ikuhara, Chem. Mater., 2012, 24, 3363.

14 D. S. Lee, S. J. Jeong, M. S. Kim and J. H. Koh, J. Appl. Phys., 2012, 112, 124109.

15 C. Groh, D. J. Franzbach, W. Jo, K. G. Webber, J. Kling, L. A. Schmitt, H. J. Kleebe, S. J. Jeong, J. S. Lee and J. Rodel, Adv. Funct. Mater., 2014, 24, 356.

16 C. Groh, W. Jo and J. Rödel, J. Am. Ceram. Soc., 2014, 97, 1465.

17 N. H. Khansur, C. Groh, W. Jo, C. Reinhard, J. A. Kimpton, K. G. Webber and J. E. Daniels, J. Appl. Phys., 2014, 115, 124108.

18 H. Zhang, C. Groh, Q. Zhang, W. Jo, K. G. Webber and J. Rödel, Adv. Electron. Mater., 2015, 1500018.

19 S. V. Kalinin, A. N. Morozovska, L. Q. Chen and B. J. Rodriguez, Rep. Prog. Phys., 2010, 73, 056502.

20 V. V. Shvartsman and A. L. Kholkin, in Multifunctional polycrystalline ferroelectric materials. Processing and properties, ed. L. Pardo and J. Ricote, Springer, Dordrecht, New York, USA, 2011, pp. 409-468.

21 I. Horcas, R. Fernandez, J. M. Gomez-Rodriguez, J. Colchero, J. Gomez-Herrero and A. M. Baro, Rev. Sci. Instrum., 2007, 78, 013705.

22 A. Kumar, Y. Ehara, A. Wada, H. Funakubo, F. Griggio, S. Trolier-McKinstry, S. Jesse and S. V. Kalinin, J. Appl. Phys., 2012, 112, 052021.

23 I. K. Bdikin, J. A. Perez, I. Coondoo, A. M. R. Senos, P. Q. Mantas and A. L. Kholkin, J. Appl. Phys., 2011, 110, 052003.

24 X. Y. Liu, Y. M. Liu, S. Takekawa, K. Kitamura, F. S. Ohuchi and J. Y. Li, J. Appl. Phys., 2009, 106, 124106.

25 Y. Kim, W. Kim, H. Choi, S. Hong, H. Ko, H. Lee and K. No, Appl. Phys. Lett., 2010, 96, 012908.

26 R. O. Duda and P. E. Hart, Pattern classification and scene analysis, Wiley, New York, USA, 1973, vol. 3.

27 Advances in knowledge discovery and data mining, ed. U. M. Fayyad, G. Piatetsky-Shapiro, P. Smyth and R. Uthurusamy, AAAI/MIT Press, Cambridge, USA, 1996.

28 E. Strelcov, A. Belianinov, Y.-H. Hsieh, S. Jesse, A. P. Baddorf, Y. H. Chu and S. V. Kalinin, ACS Nano, 2014, 8, 6449.

29 C. Ma, H. Guo, S. P. Beckman and X. Tan, Phys. Rev. Lett., 2012, 109, 107602.

30 N. T. Tsou, P. R. Potnis and J. E. Huber, Phys. Rev. B: Condens. Matter, 2011, 83, 184120. 
31 V. V. Shvartsman, B. Dkhil and A. L. Kholkin, Annu. Rev. Mater. Res., 2013, 43, 423.

32 A. Kholkin, A. Morozovska, D. Kiselev, I. Bdikin, B. Rodriguez, P. P. Wu, A. Bokov, Z. G. Ye, B. Dkhil, L. Q. Chen, M. Kosec and S. V. Kalinin, Adv. Funct. Mater., 2011, 21, 1977.

33 G. Xu, P. M. Gehring, C. Stock and K. Conlon, Phase Transitions, 2006, 79, 135.
34 V. V. Shvartsman, J. Dec, T. Lukasiewicz, A. L. Kholkin and W. Kleemann, Ferroelectrics, 2008, 373, 77.

35 V. V. Shvartsman, A. L. Kholkin, I. P. Raevski, S. I. Raevskaya, F. I. Savenko and A. S. Emelyanov, J. Appl. Phys., 2013, 113, 187208.

36 B. J. Rodriguez, S. Jesse, A. N. Morozovska, S. V. Svechnikov, D. A. Kiselev, A. L. Kholkin, A. A. Bokov, Z. G. Ye and S. V. Kalinin, J. Appl. Phys., 2010, 108, 042006. 